Relations industrielles

Industrial Relations

\title{
Déclaration de principes de la C.T.C.C.
}

Volume 7, numéro 1-2, décembre 1951, mars 1952

URI : https://id.erudit.org/iderudit/1023082ar

DOI : https://doi.org/10.7202/1023082ar

Aller au sommaire du numéro

Éditeur(s)

Département des relations industrielles de l'Université Laval

ISSN

0034-379X (imprimé)

1703-8138 (numérique)

Découvrir la revue

Citer ce document

(1951). Déclaration de principes de la C.T.C.C. Relations industrielles / Industrial Relations, 7(1-2), 63-65. https://doi.org/10.7202/1023082ar

Tous droits réservés @ C Département des relations industrielles de l’Université Laval, 1952
Ce document est protégé par la loi sur le droit d'auteur. L’utilisation des services d'Érudit (y compris la reproduction) est assujettie à sa politique d'utilisation que vous pouvez consulter en ligne.

https://apropos.erudit.org/fr/usagers/politique-dutilisation/ 
459-Comment les citoyens choisissent-ils consciencieusement les gouvernants?

En votant pour le candidat qu'ils jugent le plus capable d'assurer le bien commun de tous les citoyens.

460-Qu'est-ce que le quatrième commandement de Dieu ordonne aux gouvernants? Le quatrième commandement de Dieu ordonne aux gouvernants d'assurer le bien commun de tous les citoyens.

461-Qu'est-ce que le bien commun temporel de tous les citoyens?

C'est le plus grand bien-être possible en cette vie, pour l'âme et pour le corps, grâce à l'union et à la coordination des efforts de tous. citoyens?

462-Que doivent faire les gouvernants pour assurer le bien commun de tous les

Respecter les droits de tous, faire des lois justes, bien administrer la justice et répartir équitablement les impôts, les octrois et les charges publiques.

463-Quels sont les droits que l'Etat doit respecter?

Les droits supérieurs de Dieu et de l'Eglise catholique, les droits de la famille et de la personne humaine, les droits des sociétés particulières et les droits des autres pays.

\section{DECLARATION DE PRINCIPES DE LA C.T.C.C.}

\section{Caractère et but}

La Confédération des Travailleurs Catholiques du Canada est une organisation syndicale démocratique et libre. Elle est nationale et elle s'inspire dans ses principes et son action de la doctrine sociale de l'Eglise qu'elle reconnaît comme la seule capable d'assurer l'ordre social.

Elle a pour but de promouvoir les intérêts professionnels, économiques, sociaux et moraux des travailleurs du Canada.

Elle entend assurer la promotion individuelle et collective des travailleurs en favorisant leur développement physique, intellectuel et moral et en créant pour tous des conditions économiques et sociales telles qu'ils puissent vivre d'une façon humaine et chrétienne. Elle veut contribuer à l'établissement de relations ordonnées entre employeurs et employés, selon la vérité, la justice et la charité. Parmi ses objectifs immédiats dans ce domaine, elle veut assurer le plein exercice du droit naturel d'association et elle préconise les conventions collectives, les mesures de sécurité sociale et une saine législation du travail.

\section{SYNDICAT PROFESSIONNEL}

Le Syndicat est, pour les travailleurs, le moyen le plus efficace de défendre et de promouvoir leurs intérêts professionnels. En conséquence, on doit reconnaître à tous les travailleurs sans distinction le droit d'association et leur en faciliter l'exercice au besoin par des mesures de sécurité syndicale. Le Syndicat professionnel est, pour les travailleurs, l'organisme normal de négociations, de représentation, de participation et de collaboration sur tous les plans de l'entreprise, de la profession et de l'économie nationale.

Structure de la C.T.C.C.

Un Syndicat de travailleurs ne pent être admis au sein de la C.T.C.C. s'il n'est d'abord affilié à sa fédération professionnelle et au Conseil Central de sa région ou de sa localité. L'affiliation à tout autre organisme syndical constitué par les corps affiliés n'a aucun caractère obligatoire.

Aussi longtemps que sa charte d'affiliation est maintenue, tout corps affilié adhère à la présente déclaration de principes et est tenu d'observer les statuts et règlements de la C.T.C.C. 


\section{Personne humaine et bien commun}

Le régime économique actuel prédominant dans notre pays déprécie les valeurs humaines et spirituelles. D'après ce régime, la recherche de l'intérêt individuel procurerait automatiquement le bien général. Il s'ensuit le mépris de la dignité humaine, des légitimes aspirations de la personne humaine et du bien général.

L'accumulation des richesses et la concentration du pouvoir économique entre les mains d'un petit nombre au détriment du bien commun ont été la conséquence d'un système qui s'est donné comme mobile primordial le profit.

C'est un devoir pour chaque citoyen de contribuer au bien commun selon les exigences de la justice sociale. D'autre part, la société est faite pour la personne humaine. Aussi faut-il que la production des biens matériels soit ordonnée à la satisfaction des besoins humains légitimes et que toutes les conditions matérielles, sociales et culturelles favorisent l'épanouissement de chaque travailleur et de sa famille en toute sécurité et liberté.

La C.T.C.C. croit à la dignité primordiale de la personne humaine et à l'égalité fondamentale de tous les êtres humains. Elle n'admet pas qu'on applique un traitement injuste à cause de la langue, de la nationalité, de la race, du sexe et de la religion.

\section{Démocratie}

La C.T.C.C. a foi dans la vraie démocratie politique, parce que c'est le système qui garantit le mieux la liberté des citoyens et leur participation aux responsabilités civiles. Elle est d'avis que notre régime démocratique ne doit pas être à la merci de quelques privilégiés qui se servent du pouvoir pour la protection de leurs privilèges et de leurs in+érêts égoïstes. La C.T.C.C. croit qu'une véritable démocratie politique ne peut se concevoir sans la démocratisation de l'économie. Elle s'oppose à toute forme de totalitarisme et d'étatisme.

\section{L'Etat}

L'Etat doit promouvoir le bien commun de la C.T.C.C. croit qu'il doit, par ses lois et leur saine application, sauvegarder les droits de chacun et favoriser le développement de groupements intermédiaires autonomes dont la contribution active est nécessaire au maintien de la paix sociale.

\section{Propruété PRIVÉE}

L'exercice du droit de propriété doit être réglé selon la nature de l'objet, suivant qu'il s'agit d'un bien d'usage personnel ou d'un bien de production.

La propriété des biens de production est grevée de charges sociales particulières qui découlent de la nature de ces biens, de leur subordination au bien commun de la société et du caractère communautaire de l'entreprise.

Sur le plan de l'entreprise également, la C.T.C.C. croit que la propriété privée doit rester la règle générale. Cependant, elle se garde bien d'identifier propriété privée et capitalisme. Elle répudie le capitalisme libéral de même que le marxisme sous toutes ses formes.

L'Etat doit surveiller toute l'activité économique pour assurer la primauté de l'intérêt particulier. Certaines entreprises, à cause de leur grande importance pour le bienêtre des citoyens, ou de leur tendance aux abus, ont besoin d'être suivies de plus près et contenues dans des limites justes par des interventions appropriées.

S'il y a danger pour le bien commun de laisser sous le contrôle d'intérêts privés certains services ou moyens de production, la collectivité doit en assumer la charge. La gestion de ces entreprises sera confiée, autant que possible, à des corps autonomes représentatifs de tous les intéressés.

\section{Collaboration entre les AGENTS DE PRODUCTION}

La vie économique doit être organisée de façon à assurer une collaboration étroite entre les principaux agents de la production et de la distribution des biens. Cette collaboration doit s'établir sur le plan de l'entreprise, de la profession et de l'économie en général.

Dans l'entreprise, les travailleurs doivent être considérés comme des coopérateurs à une oeuvre commune. Ils doivent s'y sentir intégrés et participer à sa gestion et à ses bénéfices. De telles réformes de structure amèneront graduellement les intéressés à constituer une communauté d'activité et d'intérêts.

La direction des entreprises doit cesser de représenter exclusivement les intérêts du capital.

Les travailleurs et les employeurs, par leurs organisations syndicales, doivent se rejoindre au niveau de la profession en formant 
des commissions paritaires qui auront pour but de réglementer la vie professionnelle de telle façon qu'elle serve le mieux possible les intérêts de ses membres et de la société.

A l'échelle provinciale ou nationale, selon les juridictions établies, la C.T.C.C. propose la formation d'organismes appropriés où les représentants des travailleurs et des employeurs sont désignés par les organisations professionnelles intéressées et dont le rôle sera de coordonner et d'orienter la vie économique.

Enfin, la C.T.C.C. croit que le Canada, de concert avec les autres Etats, doit viser à l'organisation internationale de l'économie afin d'assurer une meilleure distribution des richesses et de garantir en même temps, par la sécurité et la stabilité économique dans le monde, la paix et l'harmonie entre les nations.

\section{LA FAMILLE}

La famille a une telle importance qu'on doit tout faire pour préserver son intégrité, garantir ses droits et assurer son plein épanouissement.

Antérieure à la société civile, dont elle est la première cellule, elle ne peut en aucune façon lui sacrifier son rôle, ses fonctions et ses prérogatives essentielles; le droit des époux à une vie conjugale normale, le droit du père de famille à pourvoir à la subsistance des siens, le droit de la mère à accomplir au foyer sa tâche de gárdienne, de ménagère et d'éducatrice; le droit des parents à élever leurs enfants et à leur assurer une instruction et une éducation dont ils gardent le contrôle; le droit à une habitation salubre et suffisamment spacieuse dont ils seront, autant que possible, propriétaires.

\section{Drott au travail}

La société doit assurer à chacun la possibilité de se procurer un emploi stable et rémunérateur, conforme à ses goûts et à ses aptitudes.

\section{Conditions DE TRavaIL}

Les conditions de travail doivent être saines tant au point de vue moral que physique. Elles doivent laisser au travailleur des loisirs suffisants pour remplir ses devoirs religieux, vivre à son foyer, participer à la vie sociale, se cultiver et se reposer.

\section{RÉMUNÉRATION DU TRAVAIL}

La rémunération du travail doit d'abord être suffisante pour satisfaire les besoins normaux d'une famille moyenne.

Elle doit tenir compte, en plus, de la nature du travail, de la compétence profes- sionnelle de la productivité, de la situation de l'entreprise et des exigences du bien commun.

La C.T.C.C. estime qu'il doit y avoir égalité de rémunération entre la main-d'oeuvre masculine et la main-d'oeuvre féminine pour un travail de valeur égale.

\section{SÉCURté SOCIALE}

L'insécurité est l'une des caractéristiques de la condition actuelle du travailleur et de sa famille. Elle résulte de l'insuffisance du revenu, de l'instabilité de l'emploi, et d'un manque de protection efficace contre les risques inhérents à la vie et au travail.

Pour corriger cette situation, la C.T.C.C. croit que l'on doit d'abord ordonner la production des biens matériels à la satisfaction des besoins humains légitimes et favoriser des mesures de sécurité sociale telles qu'une politique de plein emploi, des revenus de remplacement et de complément. Ces mesures devront respecter les droits, l'initiative et les prérogatives de chacun.

\section{MOUVEMENT COOPÉRATIF}

La C.T.C.C. voit dans le mouvement coopératif un excellent moyen d'assainissement économique et social et un complément nécessaire à l'action syndicale pour réduire le coût de la vie, humaniser et démocratiser l'économie.

\section{EPARGNe}

L'épargne est un acte de prévoyance et une garantie contre l'insécurité. La C.T.C.C. en reconnaît la nécessité et réclame pour le travailleur la possibilité d'épargner. Elle entend mettre en oeuvre tous les moyens nécessaires à cette fin.

Elle voit dans les coopératives d'épargne et de crédit et certains autres organismes de même nature des institutions aptes à faire servir l'argent des travailleurs à leur promotion. La plupart des grandes entreprises financières drainent actuellement la plus grande partie de l'épargne et contribuent au maintien de la dictature économique.

\section{INSTRUCTION ET CULTURE}

Trop souvent, les conditions économiques dans lesquelles vit la famille ouvrière font obstacle à la formation technique, économique, sociale et politique des travailleurs, de même qu'à leur participation à la vie culturelle. L'instruction à tous les degrés et la culture doivent être accessibles aux travailleurs. 